\title{
Dark States and Transport through Quantum Dots
}

\author{
S.B. TOOSKI* AND B.R. BuŁKA
}

Institute of Molecular Physics, Polish Academy of Sciences, M. Smoluchowskiego 17, 60-179 Poznań, Poland

We consider current through triple and quadruple quantum dot systems in an in-plane electric field and in the sequential tunneling regime. The electric field breaks symmetry of the system and can trap electron in a dark state in which current flow can completely be blocked. Consequently rotating the electric field, one can observe current oscillations and blockades due to dark state.

PACS: $71.10 .-\mathrm{w}, 33.57 .+\mathrm{c}, 73.23 .-\mathrm{b}, 73.63 . \mathrm{Kv}$

\section{Introduction}

In quantum optics dark states and coherent population trapping are well known phenomenon, in which the states are decoupled from the laser and can no longer be excited into other states (in contrast to bright states). By analogy one can consider coherent trapping in electronic transport in a system of quantum dots [1]. In the regime of high bias voltage electron tunneling processes from/to the electrodes play the same role as the laser radiation and single-electron tunneling among quantum dots corresponds intra-atomic transitions. Destructive quantum interference can lead to coherent trapping with electronic dark states. More recently, the effects of the dark states on transport through triple and quadruple dots was analyzed using the methods of the general master equation [1] and the diagonal master equation [2, 3]. In this paper we want to study dark state conditions in the presence of small and large electric field for triple and quadruple dots for different orientation of an external electric field. We restrict our considerations to the case with a single electron in the system and just one level on each dot in an infinite bias limit.

\section{Model and methods}

The schematic of a triple- and quadruple-dot system is displayed in Fig. 1. We assume that dots are coupled coherently together, which is described by the hopping matrix element $t$, and $\Gamma_{\mathrm{L}}$ and $\Gamma_{\mathrm{R}}$ are tunneling rates to the left and the right electrodes. In the presence of an electric field $\boldsymbol{E}$ the corresponding Hamiltonian of the system can be expressed as

$$
\begin{aligned}
& H_{\mathrm{QDs}}=t \sum_{i<j}\left(c_{i}^{\dagger} c_{j}+\text { H.c. }\right) \\
& +\sum_{i}\left[\varepsilon_{i}+e E r \cos (\theta+(i-1) 2 \pi / N)\right] c_{i}^{\dagger} c_{i},
\end{aligned}
$$

where electron hopping between nearest neighbor sites is described by the first term. The last term shows the effect of the electric field on the corresponding local energy

\footnotetext{
* corresponding author; e-mail: tooski@ifmpan.poznan.pl
}

$\varepsilon_{i}$ of dot. Here $e, N$, and $\theta$ denote the charge of an electron, the number of dots in the system and an angle of the electric field (displayed in Fig. 1). Since the electric field changes the local site energies, one can prepare an experiment via sweeping the gate voltage of dots.
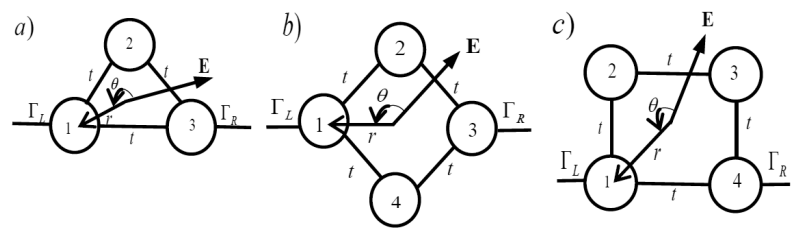

Fig. 1. Configuration for triple dots (a), symmetrically coupled quadruple dots (b), and asymmetrically coupled quadruple dots (c) in the presence of the electric field $E$.

In order to study the transport properties of the tripleand quadruple-dot system, we use the generalized master equation. Here, we give a brief consideration of the method. The density matrix $\rho(t)$ contains entries for single electron states $|i\rangle$, as well as for the empty state $|0\rangle$. Within the Born-Markov and the infinite-bias approximation, the time evolution of the density matrix is given by the generalized master equation in the Lindblad form,

$$
\begin{aligned}
& \frac{\mathrm{d} \rho}{\mathrm{d} t}=-\mathrm{i}\left[H_{\mathrm{QDs}}, \rho\right] \\
& \quad+\sum_{i=\mathrm{L}, \mathrm{R}}\left[\Gamma_{i}\left(D_{i} \rho D_{i}^{\dagger}-\frac{1}{2} D_{i}^{\dagger} D_{i} \rho-\frac{1}{2} \rho D_{i}^{\dagger} D_{i}\right)\right],
\end{aligned}
$$

where the quantum jump operators $D_{\mathrm{L}}=|L\rangle\langle 0|$ and $D_{\mathrm{R}}=|0\rangle\langle R|$ describe irreversible tunneling of electrons into and out of the quantum dot system. Here $|L\rangle$ and $|R\rangle$ are single electron states of the left and right quantum dot attached to the left and the right lead, respectively. The above master equation can then be written as $\dot{\rho}=L \rho$ with the Liouvillian $L$. The stationary properties of the system are determined by the eigenvalues and eigenvectors of $L$. In the limit of the infinite bias the current at the steady state is given by $I=e \Gamma_{\mathrm{L}} \rho_{00}(\infty) / \hbar$, where $\rho_{00}(\infty)$ is the stationary density matrix for the probability of finding the system to be empty. If $I=0$, we say that the current is blocked due to a dark state. 


\section{Current oscillation and dark state}

The application of electric field changes symmetry of the system and leads to oscillations in the current. For a special electric field orientation $\theta$, one can observe the dark-state in which current blockade occurs $(I=0)$. In the symmetric system $\left(\varepsilon_{i}=0\right.$ for any $\left.i\right)$ an exact expression for the current can be found. For the triple dot system the current can be written as

$$
\begin{aligned}
& I_{3 \mathrm{QDs}}=16 e g^{2} t^{2} \Gamma[\cos \theta+\sin (\theta+\pi / 6)]^{2} / \hbar \xi, \\
& \xi=27 g^{4}+16 t^{2} \Gamma^{2}+6 g^{2}\left(28 t^{2}+\Gamma^{2}\right) \\
& \quad-12 g t\left(6 g^{2}+\Gamma^{2}\right) \cos \theta+3 g^{2}\left(12 g^{2}+16 t^{2}+\Gamma^{2}\right) \\
& \quad \times \cos 2 \theta-36 g^{3} t \cos 3 \theta+18 g^{4} \cos 4 \theta \\
& \quad+\sqrt{3}\left[-4 g t \Gamma^{2} \sin \theta+3 g^{2}\left(\Gamma^{2} \sin 2 \theta+4 g t \sin 3 \theta\right)\right],
\end{aligned}
$$

where $\Gamma_{\mathrm{L}}=\Gamma_{\mathrm{R}}=\Gamma$ and $g=e E r$. The current for the symmetric system of four dots (Fig. 1b) can be written as

$$
\begin{aligned}
& I_{4 \mathrm{QDs}}=32 e g^{2} t^{4} \Gamma_{\mathrm{L}} \Gamma_{\mathrm{R}} \cos ^{2} \theta / \hbar v, \\
& \begin{array}{l}
v=32 g^{2} t^{4} \Gamma_{\mathrm{R}} \cos ^{2} \theta+\Gamma_{\mathrm{L}}\left(g^{4}+8 g^{2} t^{2}+8 t^{4}\right. \\
\left.\quad+g^{4} \cos 4 \theta\right)\left(16 g^{2} \cos ^{2} \theta+\Gamma_{\mathrm{R}}^{2}\right),
\end{array}
\end{aligned}
$$

whereas for the asymmetric quadruples (Fig. 1c), one gets

$$
\begin{aligned}
& I_{4 \mathrm{QDs}}=4 e g^{2} t^{2} \Gamma_{\mathrm{L}} \Gamma_{\mathrm{R}} \cos \theta \sin \theta / \hbar \beta, \\
& \begin{array}{l}
\beta=4 g^{2} t^{2} \Gamma_{\mathrm{R}} \cos \theta \sin \theta+\Gamma_{\mathrm{L}}\left[8 g^{2}\left(t^{2}+g^{2}\right) \sin 2 \theta\right. \\
\left.\quad+\Gamma_{\mathrm{R}}^{2}\left(2 t^{2}-g^{2} \cos ^{2} \theta+4 g^{2} \cos \theta \sin \theta\right)\right] .
\end{array}
\end{aligned}
$$

The results are plotted in Fig. 2. Let us first focus on the triple dot system. There can be seen current oscillations with the current blockade at some field orientations. The current blockade occurs when the electron localized in the dots 1 and 2 with the same probabilities and the electric field is perpendicular to the bond 12. Moreover, one can see that peaks of the current occur when all of the states are occupied with the same probabilities.

Next, we address the symmetrically quadruple dot systems as displayed in Fig. 2. In this case, the dark state occurs when the electric field is pointed from the dot 2 to the dot 4 or vice versa. In this situation the probability to find electron in the dot 3 is zero. This means that electron is located in the dots 1,2 , and 4 . In other words, one can say that the dot 3 is decoupled from the other dots as one expects for the dark state.

For the asymmetrically quadruple dots the dark state occurs when the electric field is pointed from the dot 1 (2) to the dot 3 (4) and vice versa (the electric field is one of the diagonal of quadruple dots). In this situation an electron is distributed between the dots 1,2 , and 3 only. The dot 4 is decoupled from the other dots and the probability to find there the electron is zero.

The above results can be understood by consideration of the interference between electron waves travelling through different paths: clockwise and anticlock-

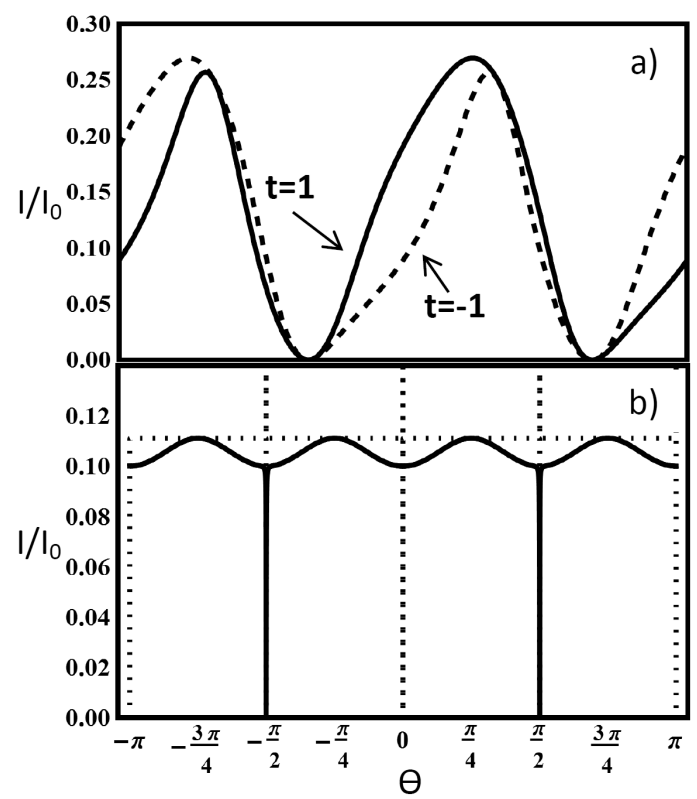

Fig. 2. Stationary current $I$ in units of $I_{0}=e \Gamma / \hbar$ versus angle $\Theta$ through the triple dots (dashed line for $t=-1 \mathrm{meV}$ and full line for $t=1 \mathrm{meV}$ ) (a), symmetrically (full line) and asymmetrically (dashed line) quadruple dots (b) for $t=1 \mathrm{meV}$. The parameters are $\Gamma=0.01 \mathrm{meV}, \varepsilon=0 \mathrm{meV}$, and $g \equiv e E r=1 \mathrm{meV}$.

wise. First we focus our attention to the triple dot system which is occupied with one electron. If we assume that at time $\tau=0$ the system is in the initial state $|\psi(0)\rangle$, then according to the time-dependent Schrödinger equation a wave function at later time $\tau$ is $|\psi(\tau)\rangle=\exp (-\mathrm{i} H \tau)|\psi(0)\rangle$, which can be expanded as $|\psi(\tau)\rangle \approx\left(1-\mathrm{i} \tau H-\tau^{2} H^{2} / 2\right)|\psi(0)\rangle$ for small times. We assume that the system is primarily in the state $|1\rangle$. The first order of the transition amplitudes from the dot 1 to 3 and the dot 2 to 3 are $a_{31}^{(1)}=\langle 3|H| 1\rangle=t$ and $a_{32}^{(1)}=\langle 3|H| 2\rangle=t$, respectively. Therefore, if the initial state is the dark state $|\psi\rangle=(|1\rangle-|2\rangle) / \sqrt{2}$, the waves in the paths from the dot 1 to 3 and the dot 2 to 3 interfere destructively at the dot 3 with a total amplitude $a_{3 \psi}^{(1)}=\langle 3|H| \psi\rangle=\left(a_{31}^{(1)}-a_{32}^{(1)}\right) / \sqrt{2}=0$.

The total second-order amplitude for the electron which is in the dark state moving from the dots 1 and 2 to the dot 3 is then $a_{3 \psi}^{(2)}=\left\langle 3\left|H^{2}\right| \psi\right\rangle=\operatorname{eErt}[\cos (\theta)+\sin (\theta+$ $\pi / 6)] / \sqrt{2}$. One can observe that this probability equals to zero and the current is blocked for $\theta=2 \pi / 3+n \pi$, $n=0,1,2, \ldots$

Now we follow the above description of the dark state conditions for the symmetrically and asymmetrically quadruple dot configurations. For both the symmetrically and asymmetrically coupled quadruple dot system, we assume that the system is primarily in the state $|1\rangle$. To the first order in $\tau$, the amplitudes for the transition from the $\operatorname{dot} 4$ to 3,1 to 4,2 to 3 , 
3 to 4 are $a_{34}^{(1)}=\langle 3|H| 4\rangle=t, a_{14}^{(1)}=\langle 1|H| 4\rangle=t$, $a_{32}^{(1)}=\langle 3|H| 2\rangle=t, a_{34}^{(1)}=\langle 3|H| 4\rangle=t$, respectively. Thus if we start with the system in the dark state $|\psi\rangle=(|4\rangle-|2\rangle) / \sqrt{2}(|\psi\rangle=(|1\rangle-|3\rangle) / \sqrt{2})$, the electron wave in the paths 123 and 143 (1234 and 14) interfere destructively at $\operatorname{dot} 3(4)$ with a total amplitude of $a_{3 \psi}^{(1)}=\langle 3|H| \psi\rangle=\left(a_{34}^{(1)}-a_{32}^{(1)}\right) / \sqrt{2}=0$ $\left(a_{4 \psi}^{(1)}=\langle 4|H| \psi\rangle=\left(a_{41}^{(1)}-a_{43}^{(1)}\right) / \sqrt{2}=0\right)$. Considering the second order transitions from dot 1 to 3 (1 to 4 ) $a_{3 \psi}^{(2)}=\left\langle 3\left|H^{2}\right| \psi\right\rangle=\operatorname{eErt} \sin (\theta)\left(a_{4 \psi}^{(2)}=\left\langle 4\left|H^{2}\right| \psi\right\rangle=\right.$ eErt $\cos (\theta))$. One can easily see that the current blockade occurs for $\theta=\pi / 2+n \pi, n=0,1,2, \ldots(\theta=$ $n \pi / 2, n=0,1,2, \ldots)$. The difference between symmetrically and asymmetrically quadruple dots can be due to different paths through the upper and lower dots: the paths 123 and 143 for the symmetric system and also 1234 and 14 for the asymmetric system.

We furthermore consider a negative value of hopping $t$. Figure 2 shows that the dark state conditions for both positive and negative $t$ are the same; however, there are some discrepancies between the current amplitude due to breaking of the electron-hole symmetry. On the other hand, for symmetrically and asymmetrically quadruple dots there is no difference between the positive and negative $t$ due to the electron-hole symmetry.

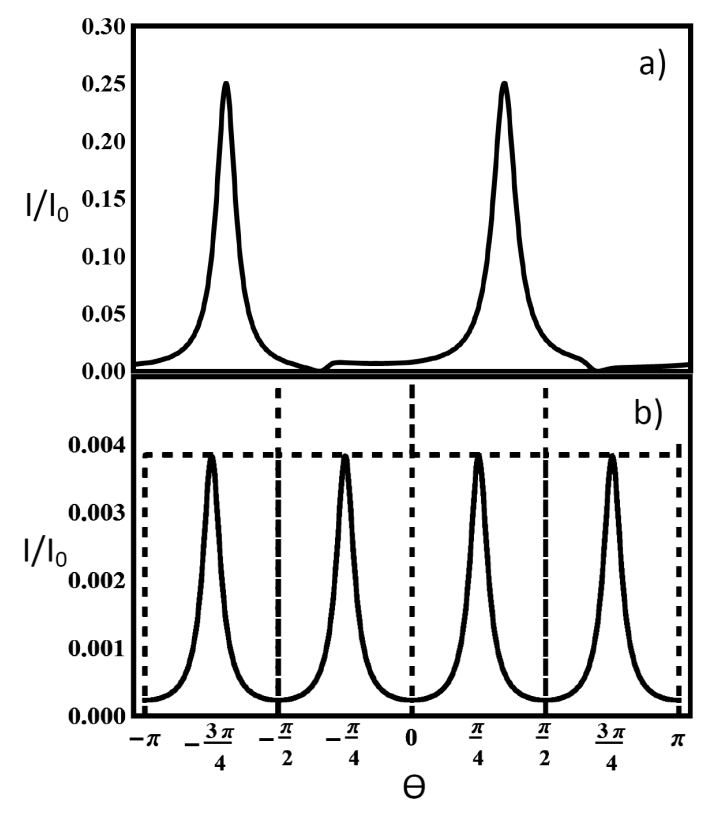

Fig. 3. Stationary current $I$ in units of $I_{0}=e \Gamma / \hbar$ versus angle $\Theta$ through the triple dots (a), symmetrically (full line) and asymmetrically quadruple dots (dashed line) (b). The parameters are $t=1 \mathrm{meV}, \Gamma=0.01 \mathrm{meV}$, $\varepsilon=0 \mathrm{meV}$, and $g \equiv e E r=8$.
Until now, we have discussed the currents for a small electric field $g \equiv e E r=1$. In the same way, we can also derive the stationary current for a very large electric $g \equiv e E r=8$ in Fig. 3. In the case of triple dots, the dark state conditions are the same for large and small electric fields. Now the current peaks are much sharper due to electron localization by high electric fields. In the case of the quadruple dot configurations, the positions of dark state are still the same; one can see very narrow dips at the same position as in Fig. 2b. Electron localization leads also in this case to sharp resonant current peaks. The current amplitudes decrease significantly with increasing electric field and it is about one hundred times smaller than the current of triple dot system. It should be mentioned that in large electric fields the currents for all of the dot configurations are not dependent on the positive and negative values of $t$.

\section{Conclusion}

The behavior of the coherently coupled triple- and quadruple-dot system in electric field studied here is governed by the coherent population trapping. One can find that a dark state, for which current blockade occurs, depends on the orientation of electric field as well as the quantum dot geometrical structures. Electric field breaks symmetry of the systems and leads to destructive quantum interference with the coherent population trapping. The electric field rotation causes current oscillations, which depend on the quantum dot geometrical structure as well as strength of the electric field.

\section{Acknowledgments}

This work was supported by the EU FP7 project: Marie Curie ITN NanoCTM and by Ministry of Science and Higher Education (Poland) from sources for science in years $2009-12$.

\section{References}

[1] C. Emary, Phys. Rev. B 76, 245319 (2007); B. Michaelis, C. Emary, C.W.J. Beenakker, Europhys. Lett. 73, 677 (2006); C. Pöltl, C. Emary, T. Brandes, Phys. Rev. B 80, 115313 (2009).

[2] W. Qiong, L. Jun, T. Ning, Z. Hao-Sheng, Chin. Phys. B 20, 020303 (2011).

[3] T. Kostyrko, B.R. Bułka, Phys. Rev. B 79, 075310 (2009); B.R. Bułka, T. Kostyrko, J. Łuczak, Phys. Rev. B 83, 035301 (2011); I. Weymann, B.R. Bułka, J. Barnas, Phys. Rev. B 83, 195302 (2011). 